\title{
AN ADJOINT METHOD FOR THE OPTIMAL BOUNDARY CONTROL OF TURBULENT FLOWS MODELED WITH THE RANS SYSTEM
}

\author{
Andrea Chierici $^{1}$, Leonardo Chirco ${ }^{1}$, Valentina Giovacchini ${ }^{1}$ and Sandro Manservisi ${ }^{1}$ \\ ${ }^{1}$ Department of Industrial Engineering DIN \\ Laboratory of Montecuccolino \\ University of Bologna \\ Via dei Colli 16, Bologna, 40136 (BO), Italy \\ Email: valentin.giovacchin2@unibo.it
}

Key words: Optimal control theory, adjoint method, boundary control, turbulence, RANS system.

\begin{abstract}
In recent years, the optimal control in fluid dynamics has gained attention for the design and the optimization of engineering devices. One of the main challenges concerns the application of the optimal control theory to turbulent flows modeled by the Reynolds averaging Navier-Stokes equations. In this work we propose the implementation of an optimal boundary control problem for the ReynoldsAveraged Navier-Stokes system closed with a two-equations turbulence model. The optimal boundary velocity is sought in order to achieve several objectives such as the enhancement of turbulence or the matching of the velocity field over a well defined domain region. The boundary where the control acts can be the main inlet section or additional injection holes placed along the domain. By minimizing the augmented Lagrangian functional we obtain the optimality system comprising the state, the adjoint, and the control equations. Furthermore, we propose numerical strategies that allow to solve the optimality system in a robust way for such a large number of unknowns.
\end{abstract}

\section{INTRODUCTION}

In last years a considerable progress has been made in mathematical analysis and optimal control problems in several research fields of fluid dynamics. Optimal control theory has been applied successfully to many fields ranging from heat transfer problems to fluid-structure interaction systems. Most of the works refer to the standard Navier-Stokes system [1,2], and the problem of turbulence is usually not taken into account because of the many difficulties arising from the numerical implementation and solution of the optimality system. Optimal control theory applied to DNS simulations for turbulent flows need a very high computational effort and it can be affordable only for simple and two-dimensional geometries [3]. To overcome this issue, in this work we consider the Reynolds averaged Navier-Stokes (RANS) system for the computation of the averaged fields. The closure of the problem is guaranteed by the implementation of a $k-\omega$ model for the estimation of eddy viscosity $\mathrm{v}_{t}$.

Distributed control strategies and feedback controls have been successfully achieved in previous works both for Navier-Stokes and RANS system $[4,5,6]$. On the other hand, few attempts of optimal boundary control for turbulent flows have been studied. In [7] the turbulence is controlled acting on the wall temperature and the buoyancy forces. In [8] a lifting function approach has been implemented in order 
to reformulate a boundary control problem into a distributed one for the RANS system closed with the Wilcox $k-\omega$ model. According to this approach, boundary controls are defined in the appropriate function boundary spaces restriction of the natural state volume spaces. However, the lifting function is solved in an extension of the domain leading to a higher computational effort. We propose instead a traditional approach for the optimal boundary control and we aim to find the optimal velocity field in the inlet sections of channels in order to achieve some objectives.

In this work we treat optimal control problems for the stationary Reynolds averaged Navier-Stokes equations closed with a $k-\omega$ model. We consider an adjoint boundary control problem for steady incompressible turbulent flows, in order to find the optimal boundary velocity that minimizes a functional computed for different objectives. In the first case, we aim at matching the velocity field in a region of the domain with desired values. In the second case, we seek to enhance the turbulence inside the channel. In both cases, goals are accomplished by the injection of fluid with a certain velocity from injection sections. We first derive, by applying the Lagrange multipliers method, the optimality system which consists of the state, adjoint and control equations and then present numerical results by using a steepest descent algorithm for the search of the local minimum of the functional.

\section{MATHEMATICAL MODEL}

In this section we first report the conservation equations modeling the physical system, then, define the optimal control problem and finally, through the Lagrange multipliers method, achieve the optimality

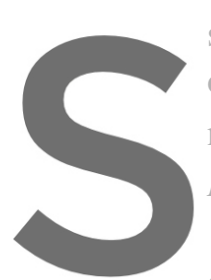
system. We use the standard notation for Lebesg

of square-integrable funct norm $\|\cdot\|_{s}$. We denote with $H^{\frac{1}{2}}$ $H^{-\frac{1}{2}}(\Gamma)$

2.1 Governing equations
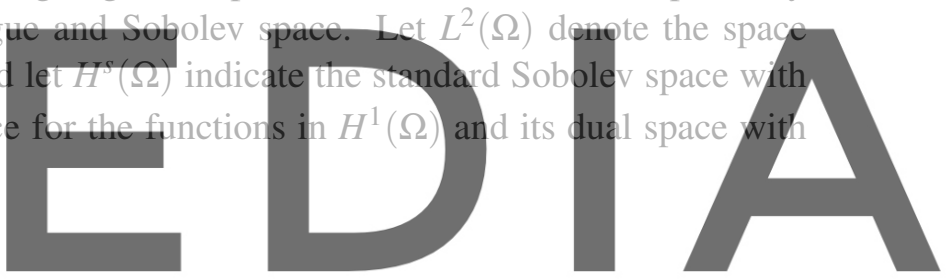

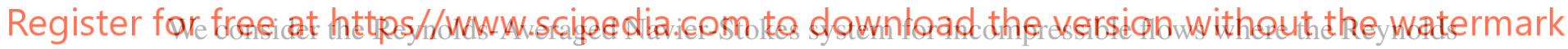
stress tensor is modeled according to the Boussinesq assumption by using mean velocity gradient components and the eddy viscosity $v_{t}$. We use the Wilcox $k$ - $w$ model to close the system and accurately evaluate $v_{t}$ [9]. The governing equations for turbulent flows in a domain $\Omega$ can be written as follows

$$
\begin{aligned}
& \nabla \cdot \mathbf{u}=0, \\
& \mathbf{u} \cdot \nabla \mathbf{u}-\nabla \cdot\left[\left(\nu+v_{t}\right) \mathbf{S}(\mathbf{u})\right]+\nabla p=0, \\
& \mathbf{u} \cdot \nabla k-\nabla \cdot\left[\left(\nu+\sigma_{k} v_{t}\right) \nabla k\right]=P_{k}-\beta_{k} k \omega, \\
& \mathbf{u} \cdot \nabla \omega-\nabla \cdot\left[\left(\nu+\sigma_{\omega} v_{t}\right) \nabla \omega\right]=c_{\omega} \frac{\omega}{k} P_{k}-\beta_{\omega} \omega^{2}+\frac{\sigma_{d}}{\omega} \nabla k \cdot \nabla \omega .
\end{aligned}
$$

The quantity $v_{t}$ is the turbulent or eddy viscosity defined in the turbulence model as

$$
v_{t}=\min \left(\frac{k}{\omega}, v_{t, \max }\right)
$$


For the interested reader the closure coefficients appearing in (3)-(5) are discussed in details in [10]. The turbulent kinetic energy source term $P_{k}$ is defined by

$$
P_{k}=\frac{v_{t}}{2} \mathbf{S}(\mathbf{u})^{2},
$$

with $\mathbf{S}(\mathbf{u})^{2}=\mathbf{S}(\mathbf{u}): \mathbf{S}(\mathbf{u})$, where $\mathbf{S}(\mathbf{u})$ is the deformation tensor

$$
\mathbf{S}(\mathbf{u})=\nabla \mathbf{u}+\nabla \mathbf{u}^{T} .
$$

We use a near-wall approach for the boundary conditions of the turbulence problem. We set the boundary conditions along the linear region at a distance $y_{d}$ from the wall, so that the value of $\omega$ remains limited in the computational domain

$$
\mathbf{u} \cdot \hat{\mathbf{n}}=0, \quad \mathbf{S}(\mathbf{u}) \cdot \hat{\mathbf{n}}=\frac{\mathbf{u} \cdot \hat{\mathbf{t}}}{y_{d}}, \quad \nabla k \cdot \hat{\mathbf{n}}=\frac{2 k}{y_{d}}, \quad \omega=\frac{2 v}{\beta_{k} y_{d}^{2}} \quad \text { on } \Gamma_{w} .
$$

\subsection{Boundary control and cost functional}

The first step to obtain the optimality system is to choose an objective for our optimal control problem. The objective functional is denoted by $\mathcal{F}(\mathbf{u}, k)$ and is described by the following expression

$$
\mathcal{F}(\mathbf{u}, k)=\frac{a}{2} \int_{\Omega_{d}}\left\|\mathbf{u}-\mathbf{u}_{d}\right\|^{2} d \Omega+\frac{b}{2} \int_{\Omega_{d}}\left\|k-k_{d}\right\|^{2} d \Omega,
$$

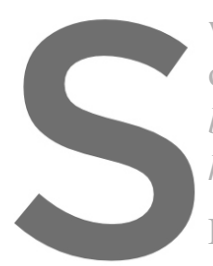

where $a$ and $b$ are weignt objective is expressed $b \neq 0$ and $a=0$ the objec $k_{d}$.
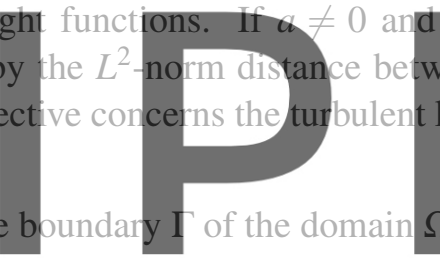

$$
\mathbf{u}=\mathbf{u}_{C} \quad \text { on } \Gamma_{C},
$$

(10)

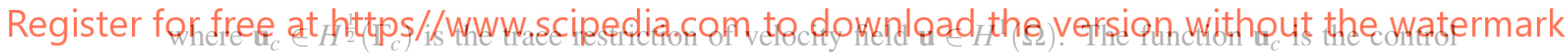
parameter that we aim to optimize in order to achieve some objectives.

In standard boundary control approaches the objective or cost functional is reformulated as

$$
\mathcal{J}\left(\mathbf{u}, k, \mathbf{u}_{c}\right)=\mathcal{F}(\mathbf{u}, k)+\frac{\lambda_{1}}{2} \int_{\Gamma_{c}}\left\|\mathbf{u}_{c}\right\|^{2} d \Gamma+\frac{\lambda_{2}}{2} \int_{\Gamma_{c}}\left\|\nabla_{s} \mathbf{u}_{c}\right\|^{2} d \Gamma,
$$

where $\nabla_{s}$ represents the surface gradient operator. The last two terms are penalization contributions introduced to limit the size of control parameter $\mathbf{u}_{c}$ and its gradient $\nabla_{s} \mathbf{u}_{c}$. In this way, the boundary control parameter is limited to Sobolev space $H^{1}\left(\Gamma_{c}\right)$, although its natural space is $H^{\frac{1}{2}}\left(\Gamma_{c}\right)$. To enforce $\mathbf{u}_{c}$ to belong to its natural space it is possible to implement a fractional norm or to adopt a lifting function approach already presented in [8]. In this work we choose to enforce stronger regularity requirements.

The values of $\lambda_{1}$ and $\lambda_{2}$ are important for the solution of the optimal control problem. If $\lambda_{2}=0$, the control variable $\mathbf{u}_{c}$ belongs to $L^{2}\left(\Gamma_{c}\right)$, i.e. in a less regular space than its natural one. In the same way, if $\lambda_{1} \rightarrow 0$, the control variable tends to belong to distributional spaces. For this reason low values of penalization coefficients may lead to convergence issues in the numerical solution of the optimality system. On the other hand, high values of $\lambda_{1}$ and $\lambda_{2}$ may result in too smooth control and poor optimization [11]. 


\subsection{Optimality system}

In order to obtain the adjoint equations and the optimality system, the augmented Lagrangian functional is written considering the cost functional $\mathcal{I}\left(\mathbf{u}, k, \mathbf{u}_{c}\right)$ and the constraints expressed in the system (15) multiplied by appropriate Lagrange multipliers. To reproduce the inequality constraint in (5), we introduce auxiliary variables and transform the inequality into an equality constraint that can be treated with standard techniques. We write condition (5) as

$$
\left(v_{t}-\frac{k}{\omega}\right)\left(v_{t}-v_{t, \max }\right)=0
$$

and we introduce two new real variables $\gamma_{1}$ and $\gamma_{2}$ defined by

$$
\begin{aligned}
& \gamma_{1}^{2}=v_{t, \text { max }}-v_{t}, \\
& \gamma_{2}^{2}=\frac{k}{\omega}-v_{t} .
\end{aligned}
$$

The condition (12) is satisfied if $v_{t}=k / \omega$ or $v_{t}=v_{t, \max }$. If $k / \omega>g, v_{t}$ must be equal to $v_{t, \max }$ in order to verify (13) and (14), since left hand side terms are always positive. We also introduce the Lagrange multiplier $\tau_{a}$ to enforce the boundary condition (10). The augmented Lagrangian functional is given by the following expression
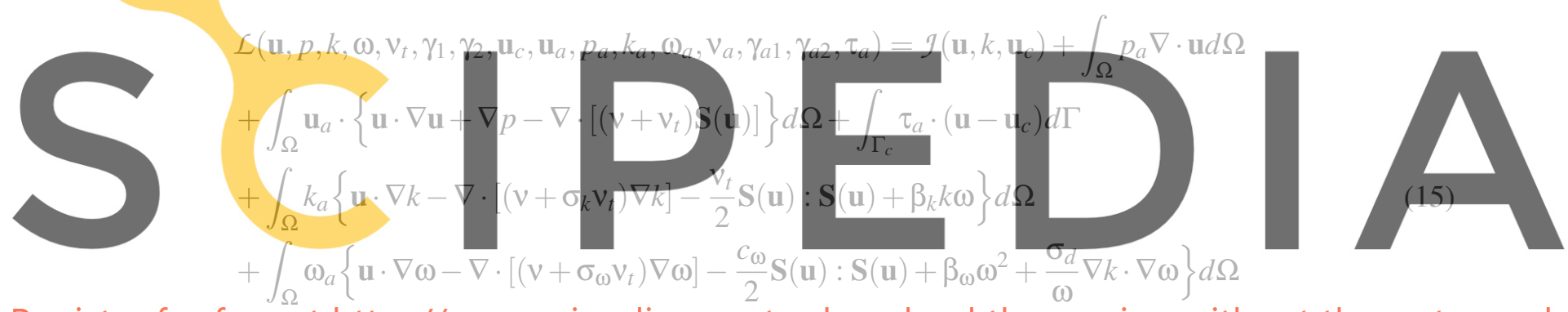

Register for free at httpsk $/$ www.scipedia.com to download the version without the watermark $+$

We use label $a$ to denote the Lagrange multipliers $\left(\mathbf{u}_{a}, p_{a}, k_{a}, \omega_{a}, v_{a}, \gamma_{a 1}, \gamma_{a 2}\right)$ of the corresponding state variables $\left(\mathbf{u}, p, k, \omega, v_{t}, \gamma_{1}, \gamma_{2}\right)$.

To obtain the optimality system we impose the first order necessary condition $\delta \mathcal{L}=0$ [11]. In the following we denote with $\delta q$ the variation of a generic function $q$ and with $(D \mathcal{L} / D q) \delta q$ the Fréchet derivative of the functional $\mathcal{L}$ in the direction $\delta q$. The stationary points of the Lagrangian functional can be found by setting to zero the Fréchet derivatives taken with respect to all the variables, since each variation is independent from the others. When the derivatives are taken with respect to the adjoint variables $\left(\mathbf{u}_{a}, p_{a}, k_{a}, \omega_{a}, v_{a}, \gamma_{a 1}, \gamma_{a 2}, \tau_{a}\right)$, the state system is recovered together with the boundary conditions. To obtain the weak form of the adjoint or dual system, we take the Fréchet derivatives with respect to the state variables $\left(\mathbf{u}, p, k, \omega, v_{t}, \gamma_{1}, \gamma_{2}\right)$.

Considering the variations $\delta p$ and $\delta \mathbf{u}$, we obtain

$$
\int_{\Omega} \nabla \cdot \mathbf{u}_{a} \delta p d \Omega-\int_{\Gamma} \mathbf{u}_{a} \cdot \hat{\mathbf{n}} \delta p d \Gamma=0, \quad \forall \delta p \in L^{2}(\Omega)
$$




$$
\begin{aligned}
& \int_{\Omega}\left\{(\delta \mathbf{u} \cdot \nabla) \mathbf{u} \cdot \mathbf{u}_{a}-(\mathbf{u} \cdot \nabla) \mathbf{u}_{a} \cdot \delta \mathbf{u}-\nabla p_{a} \cdot \delta \mathbf{u}-\nabla \cdot\left[\left(v+v_{t}\right) \mathbf{S}\left(\mathbf{u}_{a}\right)\right] \cdot \delta \mathbf{u}\right. \\
& \left.+k_{a} \nabla k \cdot \delta \mathbf{u}+\omega_{a} \nabla \omega \cdot \delta \mathbf{u}+\nabla \cdot\left[\left(k_{a} v_{t}+c_{\omega} \omega_{a}\right) \mathbf{S}(\mathbf{u})\right] \cdot \delta \mathbf{u}\right\} d \Omega= \\
& =\int_{\Gamma}\left\{-p_{a} \hat{\mathbf{n}} \cdot \delta \mathbf{u}-(\mathbf{u} \cdot \hat{\mathbf{n}})\left(\mathbf{u}_{a} \cdot \delta \mathbf{u}\right)+\left(v+v_{t}\right) \mathbf{S}(\delta \mathbf{u}) \cdot \hat{\mathbf{n}} \cdot \mathbf{u}_{a} \quad \forall \delta \mathbf{u} \in \mathbf{H}^{1}(\Omega)\right. \\
& \left.+\left(k_{a} v_{t}+c_{\omega} \omega_{a}\right) \mathbf{S}(\mathbf{u}) \cdot \delta \mathbf{u} \cdot \hat{\mathbf{n}}-\left(v+v_{t}\right) \mathbf{S}\left(\mathbf{u}_{a}\right) \cdot \delta \mathbf{u} \cdot \hat{\mathbf{n}}\right\} d \Gamma \\
& -\int_{\Gamma_{c}} \tau_{a} \cdot \delta \mathbf{u} d \Gamma
\end{aligned}
$$

The natural boundary conditions for the optimality system in strong form can be obtained setting equal to zero the surface integrals containing unknown terms or non-integrable functions. Let be $\Gamma_{c}$ the controlled surface, $\Gamma_{w}$ the walls, $\Gamma_{o}$ the outlet and $\Gamma_{c} \cup \Gamma_{w} \cup \Gamma_{o}=\Gamma$.

Over $\Gamma_{c}$, we set $\mathbf{u}_{a}=0, k_{a}=\omega_{a}=0$ and $p_{a}=0$, then we can deduce the expression of the Lagrangian multiplier $\tau_{a} \in H^{-1}\left(\Gamma_{c}\right)$

$$
\tau_{a}=-\left(\mathrm{v}+\mathrm{v}_{t}\right) \mathbf{S}\left(\mathbf{u}_{a}\right) \cdot \hat{\mathbf{n}} \quad \text { on } \Gamma_{c} .
$$

In the outlet section $\Gamma_{o}$, we have for the state variables $\mathbf{S}(\mathbf{u}) \cdot \hat{\mathbf{n}}=0$ and $p=0$. In order to enforce the integration to vanish, we have

$$
p_{a}=0, \quad\left(v+v_{t}\right) \mathbf{S}\left(\mathbf{u}_{a}\right) \cdot \hat{\mathbf{n}}=(\mathbf{u} \cdot \hat{\mathbf{n}}) \mathbf{u}_{a} \quad \text { on } \Gamma_{o} .
$$

The conditions for the state variables over $\Gamma_{w}$ are reported in (8) and therefore the boundary integral vanishes with
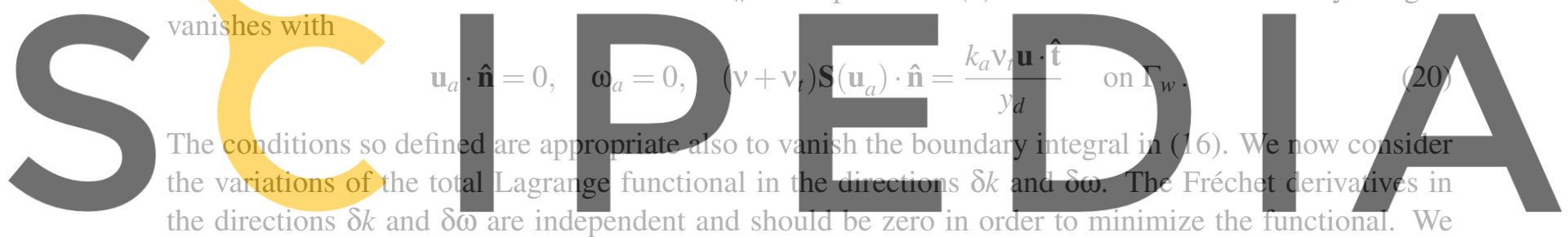

the directions $\delta k$ and $\delta \omega$ are independent and should be zero in order to 1

Register for free at https//www.scipedia.com to download the version without the watermark

$$
\begin{aligned}
\int_{\Omega} & \left\{-(\mathbf{u} \cdot \nabla) k_{a}-\nabla \cdot\left[\left(v+\sigma_{k} v_{t}\right) \nabla k_{a}\right]+\beta_{k} \omega k_{a}+b\left(k-k_{d}\right)-\frac{v_{a}\left(v_{t}-v_{t, \max }\right)+\gamma_{a 2}}{\omega}\right. \\
& \left.-\sigma_{d} \nabla \cdot\left[\frac{\omega_{a}}{\omega} \nabla \omega\right]\right\} \delta k d \Omega+\int_{\Gamma}\left[\mathbf{u} \cdot \hat{\mathbf{n}} k_{a} \delta k-\left(v+\sigma_{k} v_{t}\right) \nabla \delta k \cdot \hat{\mathbf{n}} k_{a}\right. \\
& \left.+\frac{\omega_{a}}{\omega} \sigma_{d} \nabla \omega \cdot \hat{\mathbf{n}} \delta k+\left(v+\sigma_{k} v_{t}\right) \nabla k_{a} \cdot \hat{\mathbf{n}} \delta k\right] d \Gamma=0, \quad \forall \delta k \in H^{1}(\Omega), \\
\int_{\Omega}\{ & -(\mathbf{u} \cdot \nabla) \omega_{a}-\nabla \cdot\left[\left(v+\sigma_{\omega} v_{t}\right) \nabla \omega_{a}\right]+2 \beta_{\omega} \omega \omega_{a}+\frac{k \gamma_{a 2}+k v_{a}\left(v_{t}-v_{t, \max }\right)}{\omega^{2}}+\beta_{k} k k_{a} \\
& \left.-\sigma_{d} \frac{\omega_{a} \nabla k \cdot \nabla \omega}{\omega^{2}}-\sigma_{d} \nabla \cdot\left(\frac{\omega_{a}}{\omega} \nabla k\right)\right\} \delta \omega d \Omega+\int_{\Gamma}\left[\mathbf{u} \cdot \hat{\mathbf{n}} \omega_{a} \delta \omega-\left(v+\sigma_{\omega} v_{t}\right) \nabla \delta \omega \cdot \hat{\mathbf{n}} \omega_{a}\right. \\
& \left.+\sigma_{d} \frac{\omega_{a}}{\omega} \nabla k \cdot \hat{\mathbf{n}} \delta \omega+\left(v+\sigma_{\omega} v_{t}\right) \nabla \omega_{a} \cdot \hat{\mathbf{n}} \delta \omega\right] d \Gamma=0, \quad \forall \delta \omega \in H^{1}(\Omega) .
\end{aligned}
$$

Setting equal to zero the surface integrals, we obtain the remaining boundary conditions. Over $\Gamma_{o}$, we have for the state variables $\nabla \omega \cdot \hat{\mathbf{n}}=\nabla k \cdot \hat{\mathbf{n}}=0$ which implies

$$
\left(v+\sigma_{k} v_{t}\right) \nabla k_{a} \cdot \hat{\mathbf{n}}=-\mathbf{u} \cdot \hat{\mathbf{n}} k_{a}, \quad\left(v+\sigma_{\omega} v_{t}\right) \nabla \omega_{a} \cdot \hat{\mathbf{n}}=-\mathbf{u} \cdot \hat{\mathbf{n}} \omega_{a},
$$


Over $\Gamma_{w}$, we have used the near wall approach (8) for state variables. For the adjoint variables, we obtain

$$
\omega_{a}=0, \quad \nabla k_{a} \cdot \hat{\mathbf{n}}=\frac{2 k_{a}}{y_{d}} .
$$

We now consider the variations of the total Lagrange functional in the direction $\delta v_{t}$ and integrate by parts to extract each variation from differential operators

$$
\begin{aligned}
& \int_{\Omega}[ \mathbf{S}(\mathbf{u}): \nabla \mathbf{u}_{a}+\sigma_{k} \nabla k \cdot \nabla k_{a}-\frac{k_{a}}{2} \mathbf{S}(\mathbf{u}): \mathbf{S}(\mathbf{u})+\sigma_{\omega} \nabla \omega \cdot \nabla \omega_{a}+v_{a}\left(2 v_{t}-v_{t, \max }-\frac{k}{\omega}\right) \\
&\left.\quad+\gamma_{a 1}+\gamma_{a 2}\right] \delta v_{t} d \Omega-\int_{\Gamma}\left(\mathbf{S}(\mathbf{u}) \cdot \hat{\mathbf{n}} \cdot \mathbf{u}_{a}-\sigma_{k} \nabla k \cdot \hat{\mathbf{n}}-\sigma_{\omega} \nabla \omega \cdot \hat{\mathbf{n}}\right) \delta v_{t} d \Gamma=0, \quad \forall \delta v_{t} \in L^{2}(\Omega) .
\end{aligned}
$$

The boundary conditions previously described make the surface integrals equal to zero. If we consider the variations of Lagrange functional with respect to the constraints $\delta \gamma_{1}$ and $\delta \gamma_{2}$ we obtain

$$
\begin{aligned}
& \int_{\Omega} 2 \gamma_{1} \gamma_{a 1} \delta \gamma_{1} d \Omega=0, \\
& \int_{\Omega} 2 \gamma_{2} \gamma_{a 2} \delta \gamma_{2} d \Omega=0,
\end{aligned}
$$

that are algebraic equations. If $v_{t}=k / \omega$ then $\gamma_{2}=0, \gamma_{1}>0$ and $\gamma_{a 1}=0$. In this case we can set $\gamma_{a 1}=v_{a}\left(1-v_{t}+k / \omega\right)$. If $v_{t}=v_{t, \max }$ then $\gamma_{1}=0, \gamma_{2}>0$ and $\gamma_{a 1}=0$ while for $\gamma_{a 2}$ we can set $\gamma_{a 2}=$
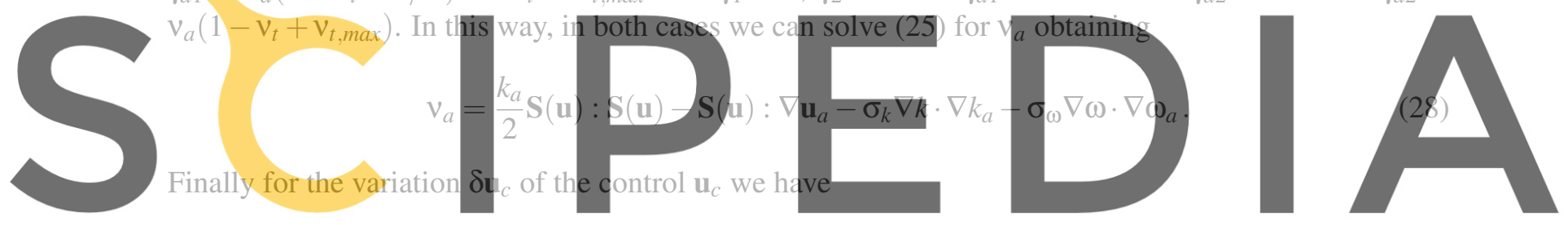

Register for free at htt $\left(\lambda_{1} \mathbf{u}_{c} \cdot \delta \mathbf{u}_{c}+\lambda_{2} \nabla_{s} \mathbf{u}_{c}: \nabla_{s} \delta \mathbf{u}_{c}-\tau_{a} \cdot \delta \mathbf{u}_{c}\right) d \Gamma=0, \quad \forall \delta \mathbf{u}_{c} \in \mathbb{H}^{1}\left(\Gamma_{c}\right.$

(29)

Register for free at htŁps//www.scipedia.com to download the version without the watermark

By substituting the equation (18) we obtain

$$
\int_{\Gamma_{c}}\left(\lambda_{1} \mathbf{u}_{c} \cdot \delta \mathbf{u}_{c}+\lambda_{2} \nabla_{s} \mathbf{u}_{c}: \nabla_{s} \delta \mathbf{u}_{c}+\left(v+v_{t}\right) \mathbf{S}\left(\mathbf{u}_{a}\right) \cdot \hat{\mathbf{n}} \cdot \delta \mathbf{u}_{c}\right) d \Gamma=0 .
$$

If $\lambda_{2}=0$, equation (30) simplifies in the following algebraic expression

$$
\mathbf{u}_{\mathbf{c}}=-\frac{\left(v+v_{t}\right)}{\lambda_{1}} \mathbf{S}\left(\mathbf{u}_{a}\right) \cdot \hat{\mathbf{n}} \text { on } \Gamma_{c} .
$$

The boundary control $\mathbf{u}_{c}$ on the boundary $\Gamma_{c}$ depends on the adjoint velocity surface normal gradient $\left(\nabla \mathbf{u}_{a}+\nabla \mathbf{u}_{a}^{T}\right) \cdot \hat{\mathbf{n}}$, on eddy viscosity $v_{t}$ and on regularization parameters $\lambda_{1}$ and $\lambda_{2}$.

The variations with respect to the adjoint variables give back the original state system (1)-(5). In the case one might be interested in a finite volume discretization it is necessary to recover the strong form of the adjoint system

$$
\nabla \cdot \mathbf{u}_{a}=0
$$




$$
\begin{gathered}
\begin{aligned}
(\mathbf{u} \cdot \nabla) \mathbf{u}_{a}-(\nabla \mathbf{u})^{T} \cdot \mathbf{u}_{a}=-\nabla p_{a}-\nabla \cdot\left[\left(v+v_{t}\right) \mathbf{S}\left(\mathbf{u}_{a}\right)\right]+k_{a} \nabla k+\omega_{a} \nabla \omega \\
+\nabla \cdot\left[\left(k_{a} v_{t}+c_{\omega} \omega_{a}\right) \mathbf{S}(\mathbf{u})\right]+a\left(\mathbf{u}-\mathbf{u}_{d}\right)
\end{aligned} \\
\begin{aligned}
(\mathbf{u} \cdot \nabla) k_{a}+\nabla \cdot\left[\left(v+\sigma_{k} v_{t}\right) \nabla k_{a}\right]= & \beta_{k} \omega k_{a}+\sigma_{d} \nabla \cdot\left(\frac{\omega_{a}}{\omega} \nabla \omega\right) \\
& +b\left(k-k_{d}\right)-\frac{\gamma_{a 2}-v_{a}\left(v_{t}-v_{t, m a x}\right)}{\omega} \\
(\mathbf{u} \cdot \nabla) \omega_{a} \nabla \cdot\left[\left(v+\sigma_{\omega} v_{t}\right) \nabla \omega_{a}\right]= & 2 \beta_{\omega} \omega \omega_{a}-\sigma_{d} \frac{\omega_{a}}{\omega^{2}} \nabla k \cdot \nabla \omega-\sigma_{d} \nabla \cdot\left(\frac{\omega_{a}}{\omega} \nabla k\right) \\
& +\frac{v_{a} k\left(v_{t}-v_{t, \max }\right)+\gamma_{a 2} k}{\omega^{2}}+\beta_{k} k k_{a}
\end{aligned} \\
v_{a}=\frac{k_{a}}{2} \mathbf{S}(\mathbf{u}): \mathbf{S}(\mathbf{u})-\mathbf{S}(\mathbf{u}): \nabla \mathbf{u}_{a}-\sigma_{k} \nabla k \cdot \nabla k_{a}-\sigma_{\omega} \nabla \omega \cdot \nabla \omega_{a} .
\end{gathered}
$$

The optimality system consists of the state system (1)-(5), the adjoint system (32)-(36), the optimality condition (30) and it is completed with the appropriate boundary conditions reported in Table 1.

Table 1: Boundary conditions with a near wall approach for the state variables $(\mathbf{u}, p, k, \omega)$ and for the adjoint variables $\left(\mathbf{u}_{a}, p_{a}, k_{a}, \omega_{a}\right)$.
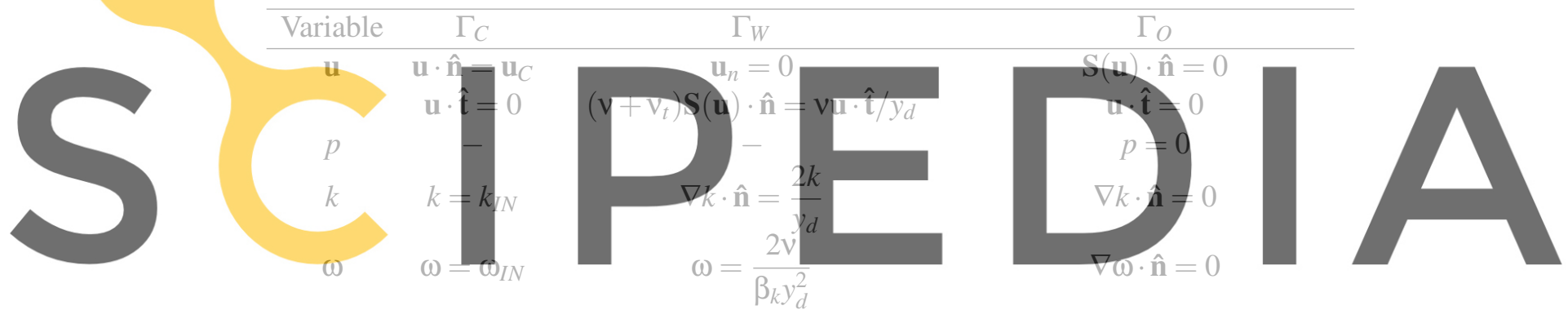

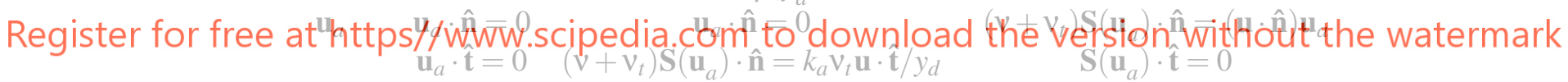

$\begin{array}{cccc}p_{a} & & & p_{a}=0 \\ k_{a} & k_{a}=0 & \nabla k_{a} \cdot \hat{\mathbf{n}}=2 k_{a} / y_{d} & \left(\boldsymbol{v}+\sigma_{k} v_{t}\right) \nabla k_{a} \cdot \hat{\mathbf{n}}=-\mathbf{u} \cdot \hat{\mathbf{n}} k_{a} \\ \omega_{a} & \omega_{a}=0 & \omega_{a}=0 & \left(\boldsymbol{v}+\sigma_{\omega} v_{t}\right) \nabla \omega_{a} \cdot \hat{\mathbf{n}}=-\mathbf{u} \cdot \hat{\mathbf{n}} \omega_{a}\end{array}$

A velocity matching objective is easier to be achieved since in the adjoint velocity equation (33) the source term $a\left(\mathbf{u}-\mathbf{u}_{d}\right)$ appears and acts directly on $\mathbf{u}_{a}$, which appears in its turn in the optimality condition (30). For this reason, a velocity matching objective is easier to be achieved. If a turbulent kinetic energy objective is considered, the source term, $b\left(k-k_{d}\right)$, acts on $k_{a}$ in (34) and through the adjoint $\mathbf{u}_{a}$ affects the velocity field to obtain an enhancement or reduction of turbulent kinetic energy. In this case the control is much more complex and the optimality system is strongly coupled.

Due to its strong nonlinearity, the optimality system cannot be solved with one-shot techniques. We use the following steepest descent algorithm:

1. Setup 
a) Set an initial value for the control $\mathbf{u}_{c}^{0}$ and an initial state $\left(\mathbf{u}^{0}, p^{0}, k^{0}, \omega^{0}, v_{t}^{0}\right)$ solution of the state system (1)-(5)

b) Compute the functional $g^{0}$ in (11)

c) Set $\Delta \mathbf{u}_{c}^{0}=0, r^{0}=1$

2. Solve the adjoint system (32)-(36) for the adjoint variables $\left(\mathbf{u}_{a}^{i}, p_{a}^{i}, k_{a}^{i}, \omega_{a}^{i}, v_{a}^{i}\right)$

3. Set the control update $\Delta \mathbf{u}_{c}^{i}=\mathbf{u}_{c}$ by solving (30) and compute $\mathbf{u}_{c}^{i}=\mathbf{u}_{c}^{i-1}+r^{i, j} \Delta \mathbf{u}_{c}^{i}$

4. Solve (1)-(5) for the state variables $\left(\mathbf{u}^{i, j}, p^{i, j}, k^{i, j}, \omega^{i, j}, v_{t}^{i, j}\right)$

5. Compute the functional $g^{i, j}$ in (11)

a) if $\left\|g^{i, j}-g^{i, j-1}\right\| / \mathfrak{g}^{i, j-1}<$ toll convergence of the optimal control problem is reached

b) else if $g^{i, j}>g^{i, j-1}$ set $r^{i, j+1}=0.5 r^{i, j}$ and return to step 3 .

c) else if $g^{i, j}<g^{i, j-1}$ set $r^{i, j+1}=r^{0}$ and return to step 2 .

\section{Numerical Results}
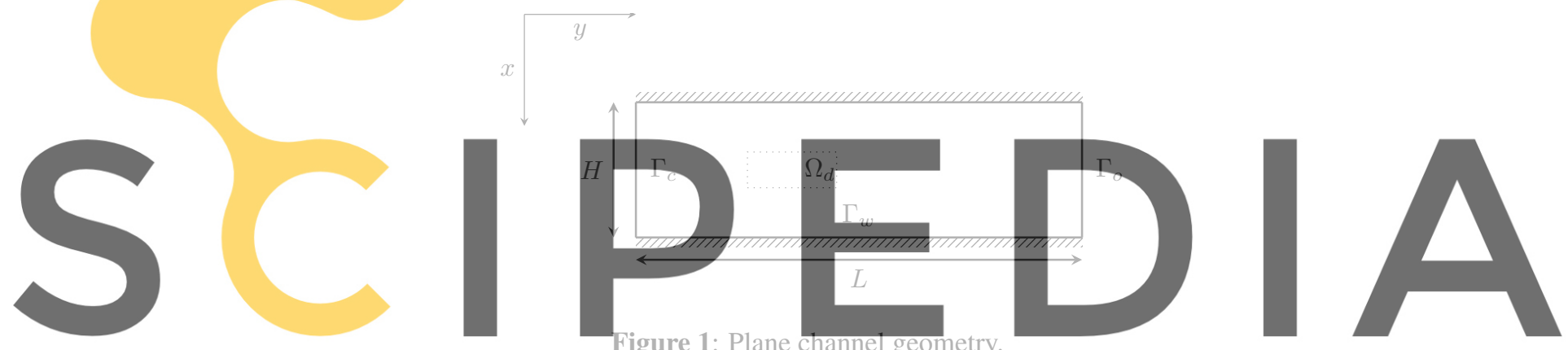

Register for free at https//www.scipedia.com to download the version without the watermark In this section, we solve the optimality system in a two-dimensional geometry for velocity matching and turbulence enhancement cases. We use a finite element discretization of the optimality system written in weak form, replacing the infinite-dimensional spaces with appropriate finite-dimensional spaces. We use a differentiate-then-discretize approach since we apply the finite element approximation after computing the Fréchet differentials. We use quadratic finite elements for all the variables but the pressure, for which we use linear finite elements. The steepest descent algorithm has been implemented in the finite element code FEMuS [12] and the PETSc libraries are used to handle the algebraic solver of the system.

The geometry we have tested is the plane channel with $H=0.1 \mathrm{~m}$ and $L=0.0303 \mathrm{~m}$ reported in Figure 1 . The rectangular domain $\Omega$ is defined as $\Omega=\{(x, y): x \in[0,0.0303], y \in[-0.05,0.05]\}$. The fluid flows from the controlled boundary $\Gamma_{c}$, on the left, to the outflow section $\Gamma_{o}$, on the right, while the region of boundary $\Gamma_{w}$ represents the walls where we have set the near-wall boundary conditions as reported in Table 1. The $y$-axis is set along the flow direction while the $x$-axis is along the transverse one. The bulk mesh consists of 15655 nodes with mesh refinement near wall boundaries. We have considered liquid lead with density $\rho=10340 \mathrm{~kg} / \mathrm{m}^{3}$ and dynamic viscosity $\mu=0.00181 \mathrm{~Pa} \cdot \mathrm{s}$. 


\subsection{Test 1. Velocity Matching Case}

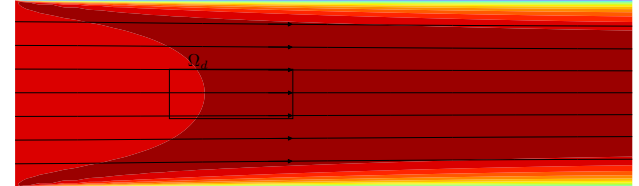

$\|\mathbf{u}\|[m / s]$

a)

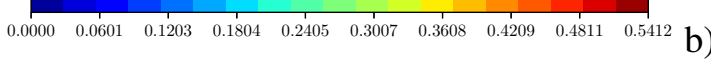

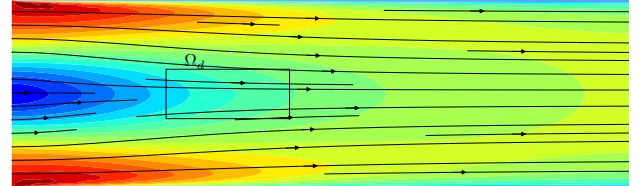

$\|\mathbf{u}\|[m / s]$

Figure 2: Test 1: Velocity Matching Case. Isosurface contours and streamlines of the velocity for the uncontrolled case (a) and for $\lambda_{1}=10^{-8}$ and $\lambda_{2}=10^{-4}(\mathrm{~b})$.
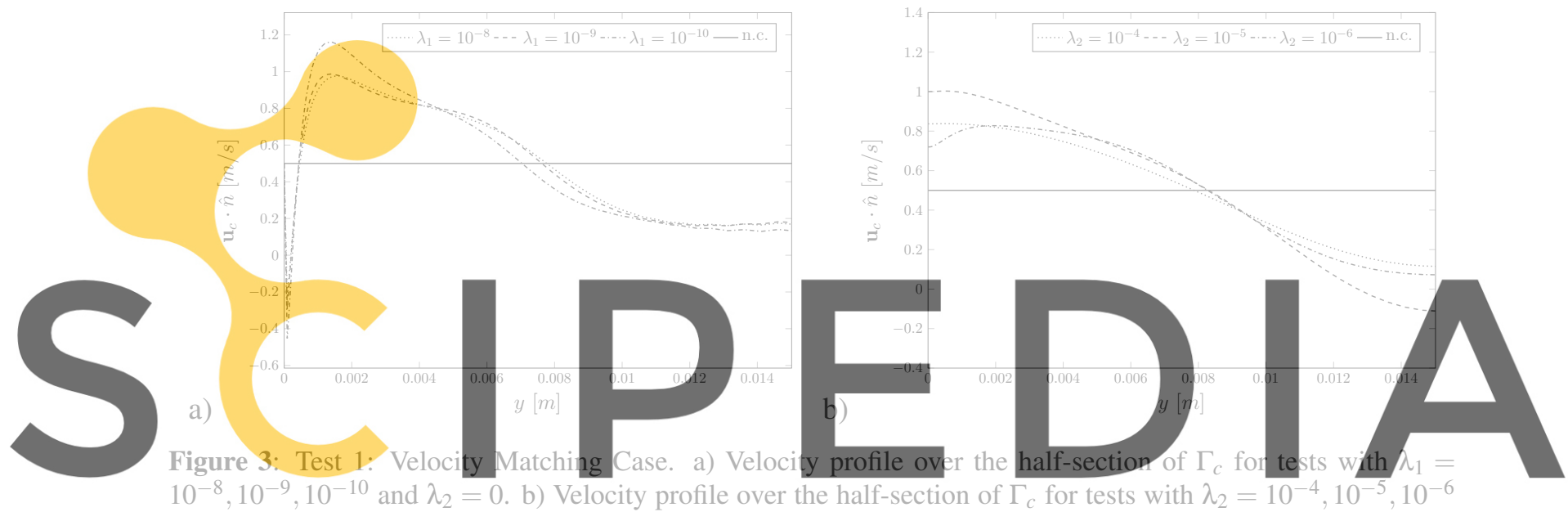

$10^{-8}, 10^{-9}, 10^{-10}$
and $\lambda_{1}=10^{-9}$

Register for free at https//www.scipedia.com to download the version without the watermark

Let be $\Omega_{d}=\{(x, y): x \in[0.01115,0.01915], y \in[-0.025,-0.005]\}$ the region where we aim to minimize the objective functional with an objective on velocity field, therefore with $a=1$ and $b=0$

$$
\mathcal{J}\left(\mathbf{u}, \mathbf{u}_{c}\right)=\frac{a}{2} \int_{\Omega_{d}}\left\|\mathbf{u}-\mathbf{u}_{d}\right\|^{2} d \Omega+\frac{\lambda_{1}}{2} \int_{\Gamma_{c}}\left\|\mathbf{u}_{c}\right\|^{2} d \Gamma+\frac{\lambda_{2}}{2} \int_{\Gamma_{c}}\left\|\nabla_{s} \mathbf{u}_{c}\right\|^{2} d \Gamma
$$

We have performed several tests varying the regularization parameters $\lambda_{1}=10^{-8}, 10^{-9}, 10^{-10}$ and $\lambda_{2}=$ $10^{-4}, 10^{-5}, 10^{-6}$.

In Figure 2 a) we report the velocity profile over the domain $\Omega$ at the reference state. We have set a constant velocity profile $\mathbf{u}_{c}=(0,0.5 \mathrm{~m} / \mathrm{s})$ on the boundary $\Gamma_{c}$ as initial value for the control, resulting in $R e=u H \rho / \mu=87000$. Over $\Omega_{d}$, the velocity field assumes a maximum value of $0.5412 \mathrm{~m} / \mathrm{s}$. These simulations aim to control the distance expressed in $L^{2}$-norm between the velocity field and a target value. In this test we aim to reduce the velocity over $\Omega_{d}$ with respect to the reference case, therefore we set $\mathbf{u}_{d}=(0,0.3 \mathrm{~m} / \mathrm{s})$. In Figure $\left.2 \mathrm{~b}\right)$ the velocity over the domain $\Omega$ is reported for the optimal solution corresponding to $\lambda_{1}=10^{-8}$ and $\lambda_{2}=10^{-4}$. Over $\Omega_{d}$, the velocity assumes values around $0.3-0.4 \mathrm{~m} / \mathrm{s}$. 
Table 2: Test 1: Velocity Matching Case. Objective functionals with $\lambda_{2}=0$ and different $\lambda_{1}$ values. The reference case without control is obtained when $\lambda_{1} \rightarrow \infty$.

\begin{tabular}{ccccc}
\hline$\lambda_{1}$ & $\infty$ & $10^{-8}$ & $10^{-9}$ & $10^{-10}$ \\
\hline$g \cdot 10^{6}$ & 4.26025 & 1.93769 & 1.70594 & 1.00159 \\
\hline
\end{tabular}

Table 3: Test 1: Velocity Matching Case. Objective functional with $\lambda_{1}=10^{-9}$ and different $\lambda_{2}$ values.

\begin{tabular}{cccc}
\hline$\lambda_{2}$ & $10^{-4}$ & $10^{-5}$ & $10^{-6}$ \\
\hline$g \cdot 10^{6}$ & 4.08514 & 1.04643 & 1.00159 \\
\hline
\end{tabular}

To analyze the influence of the regularization parameter $\lambda_{1}$ on the optimal solution, we show in Figure 3 a) the results with $\lambda_{2}=0$ for different values of $\lambda_{1}$. When $\lambda_{1}$ is smaller, the control acts stronger and achieves low values of the objective functional, as the reader can see in Table 2. For the lowest considered value of $\lambda_{1}$, the optimization gives a $77 \%$ functional reduction.

In Figure $3 \mathrm{~b}$ ), we show the influence of the solution on the regularization parameter $\lambda_{2}$. We set $\lambda_{1}=10^{-9}$ and we consider $\lambda_{2}=10^{-4}, 10^{-5}, 10^{-6}$. Figure $3 \mathrm{~b}$ ) evidences that the highest considered value of $\lambda_{2}$ brings to the smoothest solution and to the poorest functional reduction (around 4\%), as reported in Table 3. When low values of $\lambda_{2}$ are considered, the boundary control parameter is closed to the solution obtained with $\lambda_{2}=0$ and brings to the strongest functional reductio

3.2 Test 2. Turbulence Enhancement Case

Let be $\Omega_{d}=\{(x, y): x \in[0.01115,0.01915], y \in[-0.025,-0.0$
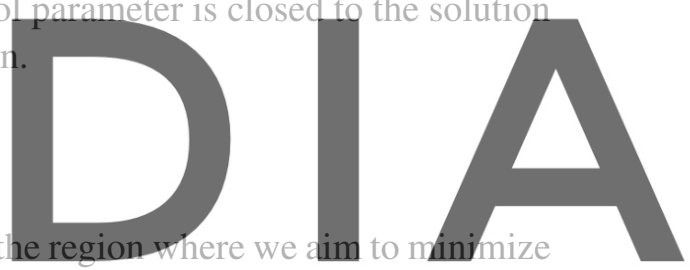

the objective functional with an objective on turbulent kinetic energy, therefore with $a=0$ and $b=1$ in

Register forffree at https//www.scipedia.com to download the version without the watermark

$$
g\left(k, \mathbf{u}_{c}\right)=\frac{b}{2} \int_{\Omega_{d}}\left|k-k_{d}\right|^{2} d \Omega+\frac{\lambda_{1}}{2} \int_{\Gamma_{c}}\left\|\mathbf{u}_{c}\right\|^{2} d \Gamma+\frac{\lambda_{2}}{2} \int_{\Gamma_{c}}\left\|\nabla_{s} \mathbf{u}_{c}\right\|^{2} d \Gamma
$$

In Figure 4 a) we report the turbulent kinetic energy profile over the domain $\Omega$ at the reference state. Also in this case, we have set a constant velocity profile $\mathbf{u}_{c}=(0,0.5 \mathrm{~m} / \mathrm{s})$ on the boundary $\Gamma_{c}$ as initial value for the control. Over $\Omega_{d}$, the turbulent kinetic energy assumes values in the order of magnitude about $10^{-4} \mathrm{~m}^{2} / \mathrm{s}^{2}$. In order to enhance the turbulence inside the channel, we aim to minimize the distance expressed in $L^{2}$-norm between the turbulent kinetic energy and a target value, i.e. $k_{d}=0.01 \mathrm{~m}^{2} / \mathrm{s}^{2}$. In Figure $4 \mathrm{~b}$ ) the turbulent kinetic energy over the domain $\Omega$ is reported for the optimal solution corresponding to $\lambda_{1}=10^{-13}$ and $\lambda_{2}=10^{-6}$. Over $\Omega_{d}$, the turbulent kinetic energy assumes values around

Table 4: Test 2: Turbulence enhancement case. Objective functionals with $\lambda_{1}=10^{-13}$ and different $\lambda_{2}$ values.

\begin{tabular}{ccccc}
\hline$\lambda_{2}$ & $\infty$ & $5 \cdot 10^{-5}$ & $10^{-5}$ & $5 \cdot 10^{-6}$ \\
\hline $\mathcal{g} \cdot 10^{10}$ & 82.7304 & 19.1531 & 12.2519 & 4.55217 \\
\hline
\end{tabular}




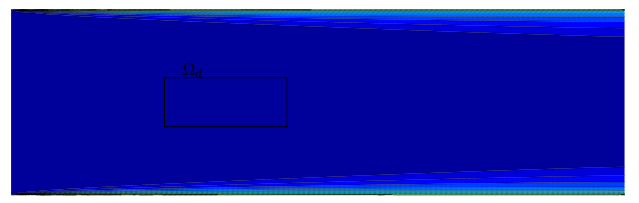

$k\left[m^{2} / s^{2}\right]$

a)

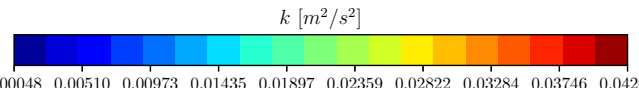

)

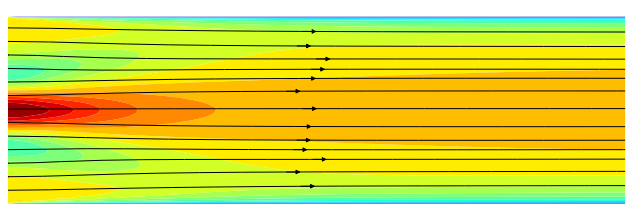

$\|\mathbf{u}\|[\mathrm{m} / \mathrm{s}]$

c)

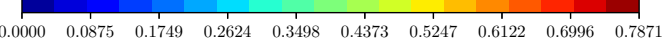

Figure 4: Test 2: Turbulence enhancement case. Isosurface contours of turbulent kinetic energy field for the uncontrolled case (a) and for $\lambda_{1}=10^{-13}$ and $\lambda_{2}=10^{-6}$ (b). Isosurface contours and streamlines of the velocity field corresponding to the optimal state (c).

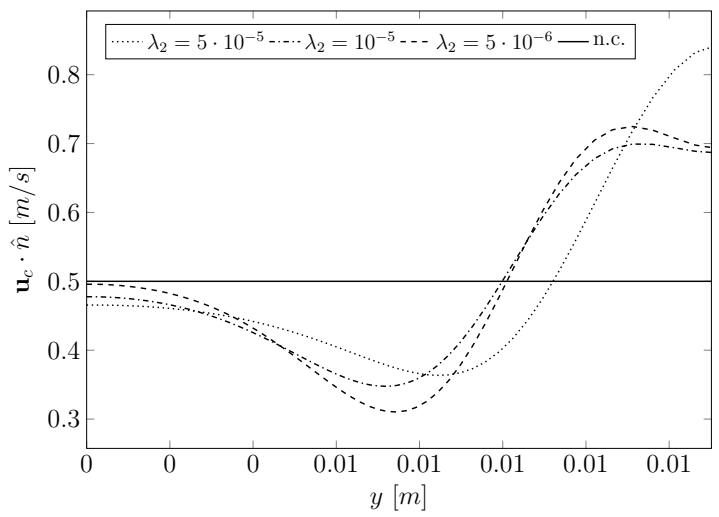

Figure 5: Test 2: Turbulence Enhancement Case. Velocity profiles along the half-section of $\Gamma_{c}$ with $\lambda_{2}=5$. $10^{-5}, 10^{-5}, 5 \cdot 10^{-6}$ and $\lambda_{1}=10^{-13}$.

$0.009-0.014 \mathrm{~m}^{2} / \mathrm{s}^{2}$. The corresponding velocity profile over the domain $\Omega$ is reported in Figure $\left.4 \mathrm{c}\right)$. The optimization algorithm leads, with this set of regularization parameters, to a velocity profile with a strong peak in the middle of the channel to maximize the turbulence over $\Omega_{d}$.

Also in this test case, we show the influence of the solution on the regularization parameter $\lambda_{2}$ in Figure 5. We set $\lambda_{1}=10^{-13}$ and we consider $\lambda_{2}=5 \cdot 10^{-5}, 10^{-5}, 5 \cdot 10^{-6}$. Figure 5 evidences that the highest considered value of $\lambda_{2}$ brings to the smoothest solution and to the poorest functional reduction (around $77 \%$ ), as reported in Table 4, while the lowest value of $\lambda_{2}$ brings to the strongest functional reduction $(95 \%)$. 


\section{Conclusions}

An adjoint-based boundary optimal control method for the Reynolds Averaged Navier-Stokes equations coupled with a two-equations turbulence model has been presented. The adjoint system has been obtained through a Lagrangian functional minimization. Proper boundary conditions have been set on the adjoint variables and a steepest descent algorithm has been implemented for the segregate solution of the optimality system. Simple test cases have been reported to show the robustness of the mathematical approach presented for different objectives and different regularization terms.

\section{REFERENCES}

[1] Gunzburger M D, Hou L and Svobodny T P 1992 SIAM journal on control and optimization 30 $167-181$

[2] Ghattas O and Bark J H 1997 Journal of Computational Physics 136 231-244

[3] Bewley T R, Moin P and Temam R 2001 Journal of Fluid Mechanics 447 179-225

[4] Manservisi S and Menghini F 2016 Computers \& Fluids 125 130-143

[5] Manservisi S and Menghini F 2016 Computers \& Mathematics with Applications 71 2389-2406

[6] Gunzburger M and Manservisi S 2000 Comput Methods Appl Mech Eng 189(3) 803-823

[7] Chirco L, Giovacchini V and Manservisi S 2020 An adjoint-based temperature boundary optimal control approach for turbulent buoyancy-driven flows Journal of Physics: Conference Series vol 1599 (IOP Publishing) p 012041

[8] Chirco L, Chierici A, Da Vià R, Giovacchini V and Manservisi S 2019 Optimal control of the wilcox turbulence model with lifting functions for flow injection and boundary control Journal of Physics: Conference Series vol 1224 (IOP Publishing) p 012006

[9] Wilcox D 1994 Turbulence Modeling for CFD (DCW Industries, Incorporated)

[10] Wilcox D C 2008 AIAA journal 46 2823-2838

[11] Gunzburger M D 2003 Perspectives in flow control and optimization vol 5 (Siam)

[12] FEMuS. URL https://github.com/FemusPlatform/femus 